\title{
Comparative in vitro antibacterial activity of ozenoxacin against Gram-positive clinical isolates
}

\author{
Rafael Canton ${ }^{1,2}$, Ian Morrissey ${ }^{3}$, Jordi Vila²,4,5, Marta Tato ${ }^{1,2}$, María García-Castillo1,2, Yuly \\ López $^{5}$, Domingo Gargallo-Viola ${ }^{6}$ \& Ilonka Zsolt*,6 \\ ${ }^{1}$ Servicio de Microbiología, Hospital Universitario Ramón y Cajal \& Instituto Ramón y Cajal de Investigación Sanitaria (IRYCIS), \\ Madrid, Spain \\ ${ }^{2}$ Red Española de Investigación en Patología Infecciosa (REIPI), Madrid, Spain \\ ${ }^{3}$ HMA Europe Sàrl, Monthey, Switzerland \\ ${ }^{4}$ Microbiology Service, Centre de Diagnòstic Biomèdic, Hospital Clinic, Barcelona, Spain \\ ${ }^{5}$ Institute of Global Health of Barcelona, Barcelona, Spain \\ ${ }^{6}$ Medical Department, Ferrer Internacional, Barcelona, Spain \\ *Author for correspondence: izsolt@ferrer.com
}

\begin{abstract}
Aim: To compare the in vitro activity of the anti-impetigo agent, ozenoxacin, and other antimicrobial agents against Gram-positive clinical isolates from skin and soft tissue infections. Materials \& methods: Isolates were collected in two studies: 1097 isolates from 49 centers during 2009-2010 and 1031 isolates from ten centers during 2014. Minimum inhibitory concentrations were determined for 18 and 11 antimicrobials in these studies, respectively, using standard broth microdilution methods. Isolates were stratified by species and methicillin susceptibility/resistance and/or levofloxacin susceptibility/nonsusceptibility status. Results: Ozenoxacin exhibited high in vitro activity against Staphylococcus aureus and coagulase-negative staphylococci isolates in both studies. Ozenoxacin was also highly active against Streptococcus pyogenes and Streptococcus agalactiae isolates. Conclusion: Ozenoxacin is a potent antimicrobial agent against staphylococci and streptococci.
\end{abstract}

First draft submitted: 4 December 2017; Accepted for publication: 16 March 2018; Published online: 10 May 2018

Keywords: antibacterial activity $\bullet$ clinical isolates $\bullet$ Gram-positive $\bullet$ MRSA $\bullet$ ozenoxacin

Impetigo is a common bacterial skin infection affecting both children and adults although it is more prevalent in children. Infection with Staphylococcus aureus or Streptococcus pyogenes causes the nonbullous type of impetigo which occurs in around $70 \%$ of cases, whereas $S$. aureus exclusively causes bullous impetigo, with the production of exfoliative toxins [1-3].

Topically administered antibacterial agents, mupirocin and fusidic acid, are commonly used to treat impetigo, although retapamulin is a more recent alternative $[4,5]$. The most recent topical option and the only compound with bactericidal properties is the nonfluorinated quinolone, ozenoxacin [6], which demonstrated clinical benefit in a recent Phase III trial [7]. The results of a second Phase III trial which is currently being published showed similar clinical and microbiological results (ClinicalTrials.gov identifier: NCT02090764) [8].

During the development of ozenoxacin, surveillance studies addressing its antimicrobial activity in comparison with other antimicrobials were conducted. In this article, the in vitro activity of ozenoxacin against Gram-positive clinical isolates recovered from skin and soft-tissue infections (SSTIs) is compared with a panel of antibacterial agents. More than 2000 isolates were collected in two worldwide studies from 2009 to 2010, and during 2014. The isolates include the causative microorganisms of impetigo, S. aureus and S. pyogenes, and coagulase-negative staphylococci which are the most common microorganisms on normal skin flora, with Staphylococcus epidermidis being the predominant species [3]. Comparisons with a wide range of antimicrobial agents were made using the minimum inhibitory concentration (MIC) value, which is also useful for assessing antimicrobial phenotypic resistance [9]. 


\section{Materials \& methods}

Study 1: evaluation of the in vitro activity of ozenoxacin \& comparative antimicrobial agents against Gram-positive clinical isolates collected during 2009 \& 2010

The in vitro activity of ozenoxacin was evaluated against Gram-positive isolates collected from 49 centers in the Czech Republic $(\mathrm{n}=3)$, Germany $(\mathrm{n}=3)$, The Netherlands $(\mathrm{n}=3)$, Romania $(\mathrm{n}=3)$, South Africa $(\mathrm{n}=3)$, Spain $(\mathrm{n}=4)$ and the USA $(\mathrm{n}=30)$. Organisms were obtained randomly from uncomplicated SSTIs (uSSTIs) and/or complicated SSTIs (cSSTIs) during 2009 and 2010. A total of 1097 isolates were collected from participating sites, with $50.0 \%$ originating from inpatients $(n=548)$ and $49.9 \%(n=547)$ from outpatients; the origin of two isolates was not recorded. Isolates were classified as $S$. aureus $(\mathrm{n}=486)$, $S$. epidermidis $(\mathrm{n}=190)$, other coagulasenegative Staphylococcus (CNS) species $(\mathrm{n}=37)$, S. pyogenes $(\mathrm{n}=217)$, Streptococcus agalactiae $(\mathrm{n}=37)$, Streptococcus pneumoniae ( $(\mathrm{n}=29)$ and Corynebacterium spp. $(\mathrm{n}=52)$. Other isolates were Micrococcus spp. $(\mathrm{n}=7)$, Lactobacillus spp. $(\mathrm{n}=7)$, Group G Streptococcus $(\mathrm{n}=19)$, Group C Streptococcus $(\mathrm{n}=9)$, and Brevibacterium spp. $(\mathrm{n}=3)$; plus Enterococcus faecalis $(\mathrm{n}=2)$, Kocuria kristinae $(\mathrm{n}=1)$ and Rothia mucilaginosa $(\mathrm{n}=1)$.

S. aureus was identified by Gram stain, catalase and DNAse production, and the staphylococcal latex agglutination test. In addition, cefoxitin susceptibility was performed to determine methicillin resistance. Identification of coagulase-negative staphylococci was performed using Gram stain, catalase and DNAse production, the staphylococcal latex agglutination test, API Identification Systems or mass spectrometry (matrix assisted laser desorption/ionization-time of flight mass spectrometry [MALDI-TOF MS], Bruker Daltonics, Bremen, Germany). Cefoxitin susceptibility was also performed. Groups A, B, C and G streptococci were identified using Gram stain, catalase production and Lancefield grouping. Identification of S. pneumoniae used Gram stain, optochin susceptibility and bile solubility. Identification of Corynebacterium spp., Micrococcus spp. and Lactobacillus spp. used Gram stain, catalase production and identification by API Identification Systems or MALDI-TOF MS.

MIC was determined by the broth microdilution method as recommended by the Clinical and Laboratory Standards Institute (CLSI) [10]. MIC $_{50}$ and $\mathrm{MIC}_{90}$ values were calculated for each antimicrobial agent.

For determination of MICs, the same range of concentrations for each antimicrobial were tested against species of Staphylococcus, Corynebacterium, Micrococcus and Lactobacillus (group 1 species); with the tested MIC range often differing for Streptococcus species. Respective tested MIC ranges for group 1 species and Streptococcus species were: ozenoxacin $(0.001-2 ; 0.001-2 \mathrm{mg} / \mathrm{l})$, amoxicillin-clavulanate $(0.03-32 ; 0.008-16 \mathrm{mg} / \mathrm{l})$, ceftriaxone $(0.03-$ 64; $0.03-64 \mathrm{mg} / \mathrm{l})$, cefuroxime (0.03-64; 0.015-16 mg/l), ciprofloxacin $(0.002-4 ; 0.03-32 \mathrm{mg} / \mathrm{l})$, clindamycin (0.03-64; 0.015-32 mg/l), daptomycin (0.015-32; 0.015-32 mg/l), erythromycin $(0.03-64 ; 0.015-32 \mathrm{mg} / \mathrm{l})$, fusidic acid (0.03-64; 0.03-64 mg/l), gentamycin $(0.03-32 ; 0.03-64 \mathrm{mg} / \mathrm{l})$, levofloxacin $(0.004-8 ; 0.03-64 \mathrm{mg} / \mathrm{l})$, linezolid (0.015-32; 0.015-32 mg/l), mupirocin (0.03-64; 0.03-64 mg/l), neomycin $(0.03-64 ; 0.03-64 \mathrm{mg} / \mathrm{l})$, penicillin G $(0.03-64 ; 0.008-16 \mathrm{mg} / \mathrm{l})$, retapamulin $(0.008-16 ; 0.008-16 \mathrm{mg} / \mathrm{l})$, tetracycline $(0.03-64 ; 0.03-$ $64 \mathrm{mg} / \mathrm{l}$ ) and vancomycin (0.03-32; 0.03-32 mg/l). Two quality-control strains (S. aureus ATCC 29213 and $S$. pneumoniae ATCC 49619) were also included in the study.

Susceptibility results were interpreted using breakpoints set by the CLSI [10,11]. Nevertheless, European Committee on Antimicrobial Susceptibility Testing (EUCAST) [12] or British Society for Antimicrobial Chemotherapy [13] breakpoints were used for different antimicrobials: staphylococci and fusidic acid [13]; S. pneumoniae and ciprofloxacin [12]; staphylococci and mupirocin [13]; and Corynebacterium species for amoxicillin-clavulanate, cefuroxime and levofloxacin [12]. No breakpoints are yet defined by the CLSI or EUCAST for ozenoxacin.

\section{Study 2: evaluation of the in vitro activity of ozenoxacin \& comparative antimicrobials against relevant clinical isolates collected during 2014}

A total of 1031 clinical isolates of $S$. aureus $(\mathrm{n}=504)$, coagulase-negative staphylococci $(\mathrm{n}=315)$, $S$. pyogenes $(\mathrm{n}=124)$ and $S$. agalactiae $(\mathrm{n}=88)$ were collected from January to December 2014 at ten participating centers located in Argentina, Brazil, Colombia, Germany, Romania, South Africa, Spain, Sweden and at two sites in the USA. Species in the coagulase-negative staphylococci group were Staphylococcus capitis $(\mathrm{n}=17)$, Staphylococcus caprae $(\mathrm{n}=4)$, Staphylococcus cohni $(\mathrm{n}=1)$, S. epidermidis $(\mathrm{n}=195)$, Staphylococcus haemolyticus $(\mathrm{n}=27)$, Staphylococcus hominis $(\mathrm{n}=12)$, Staphylococcus intermedious $(\mathrm{n}=3)$, Staphylococcus lugdunensis $(\mathrm{n}=42)$, Staphylococcus saprophyticus $(\mathrm{n}=2)$, Staphylococcus simulans $(\mathrm{n}=4)$ and Staphylococcus warneri $(\mathrm{n}=8)$. Two quality-control strains $(S$. aureus ATCC 29213 and S. pneumoniae ATCC 49619) were also included in the study.

All isolates were tested for susceptibility to amoxicillin-clavulanate, ciprofloxacin, clindamycin, erythromycin, fusidic acid, mupirocin, levofloxacin, ozenoxacin, penicillin and vancomycin using prepared dry panels (Sensititre ${ }^{\mathrm{TM}}$, 
Thermo Fisher Scientific). They were prepared using the broth microdilution method as recommended by the CLSI [10]. The range of concentrations tested were: ozenoxacin $(0.001-16 \mathrm{mg} / \mathrm{l})$, amoxicillin-clavulanate $(4 / 2-$ $8 / 2 \mathrm{mg} / \mathrm{l})$, ciprofloxacin $(0.015-16 \mathrm{mg} / \mathrm{l})$, clindamycin $(0.015-16 \mathrm{mg} / \mathrm{l})$, erythromycin $(0.015-16 \mathrm{mg} / \mathrm{l})$, fusidic acid $(0.015-16 \mathrm{mg} / \mathrm{l})$, levofloxacin $(0.015-16 \mathrm{mg} / \mathrm{l})$, mupirocin $(0.015-256 \mathrm{mg} / \mathrm{l})$, penicillin $\mathrm{G}(0.12-0.25 \mathrm{mg} / \mathrm{l})$, retapamulin $(0.015-1 \mathrm{mg} / \mathrm{l})$ and vancomycin $(0.5-2 \mathrm{mg} / \mathrm{l})$. Susceptibility results were interpreted using an approach that was similar to the previous study but using CLSI and EUCAST documents from 2014.

\section{Results}

\section{Source of isolates \& quality control results}

Isolates in study 1 were recovered from skin $(\mathrm{n}=541 ; 49.3 \%)$, wounds $(\mathrm{n}=300 ; 27.3 \%)$, abscesses $(\mathrm{n}=98 ; 8.9 \%)$, tissue $(n=45 ; 4.1 \%)$, blood $(n=40 ; 3.6 \%)$ or other $(n=73 ; 6.7 \%)$. SSTIs were further categorized as cSSTI, uSSTI or unknown. The proportion of skin isolates (expressed as a percentage of the total sample) categorized as cSSTI, uSSTI and unknown were 18.3\% $(\mathrm{n}=201), 23.4 \%(\mathrm{n}=257)$ and $7.6 \%(\mathrm{n}=83)$, respectively. Respective proportions by site of recovery were, for wound: $10.8 \%(\mathrm{n}=118), 8.2 \%(\mathrm{n}=92)$ and $8.4 \%(\mathrm{n}=90)$; for abscess: 2.6\% $(\mathrm{n}=28), 1.9 \%(\mathrm{n}=49)$ and $4.5 \%(\mathrm{n}=21)$; for tissue: $2.5 \%(\mathrm{n}=27), 1.5 \%(\mathrm{n}=2)$ and $0.2 \%(\mathrm{n}=16)$; for blood: $1.3 \%(\mathrm{n}=14 ; 1.7 \%(\mathrm{n}=7)$ and $0.6 \%(\mathrm{n}=19)$; and for other: $2.2 \%(\mathrm{n}=24), 1.5 \%(\mathrm{n}=33)$ and $3.0 \%$ $(\mathrm{n}=16)$.

Isolates in study 2 were recovered from wounds $(\mathrm{n}=410 ; 39.8 \%)$, abscesses $(\mathrm{n}=163 ; 15.8 \%)$, skin $(\mathrm{n}=20$; $1.9 \%)$, acne $(n=11 ; 1.1 \%)$, tissues $(n=8 ; 0.8 \%)$ and nonspecified SSTIs $(n=419 ; 40.6 \%)$.

All quality control results were within the quality control ranges specified by CLSI Documents M100-S20 (2011) [14] and M100-S24 (2014) [15].

\section{S. aureus}

\section{Study 1}

Table 1 shows $\mathrm{MIC}_{50}$ and $\mathrm{MIC}_{90}$ values for ozenoxacin and 18 comparator antimicrobial agents against $S$. aureus isolates, which were also stratified by methicillin (-susceptible $S$. aureus [MSSA]; -resistant $S$. aureus [MRSA]) and levofloxacin susceptibility. Ozenoxacin was highly active against the $486 \mathrm{~S}$. aureus isolates tested, with a $\mathrm{MIC}_{50}$ of $0.004 \mathrm{mg} / \mathrm{l}$ and a $\mathrm{MIC}_{90}$ of $0.25 \mathrm{mg} / \mathrm{l}$. For levofloxacin-nonsusceptible $S$. aureus isolates $(\mathrm{n}=168$; levofloxacin $\mathrm{MIC}_{50}=8 \mathrm{mg} / \mathrm{l}$ and $\mathrm{MIC}_{90}=16 \mathrm{mg} / \mathrm{l}$ ), the ozenoxacin $\mathrm{MIC}_{50}$ was $0.12 \mathrm{mg} / \mathrm{l}$ and the $\mathrm{MIC}_{90}$ was $0.5 \mathrm{mg} / \mathrm{l}$. Ozenoxacin had lower MIC values $\left(\mathrm{MIC}_{50}=0.004 \mathrm{mg} / \mathrm{l}\right.$ and $\left.\mathrm{MIC}_{90}=0.004 \mathrm{mg} / \mathrm{l}\right)$ against levofloxacin-susceptible $S$. aureus isolates $\left(\mathrm{n}=312\right.$; levofloxacin $\mathrm{MIC}_{50}=0.25 \mathrm{mg} / \mathrm{l}$ and $\mathrm{MIC}_{90}=0.25 \mathrm{mg} / \mathrm{l}$; Table 1$)$. The $\mathrm{MIC}_{90}$ of ozenoxacin $(0.25 \mathrm{mg} / \mathrm{l})$ was comparable to those of fusidic acid, mupirocin and retapamulin (all $0.25 \mathrm{mg} / \mathrm{l}$ ) against all $S$. aureus isolates tested. In contrast, ciprofloxacin $(8 \mathrm{mg} / \mathrm{l})$ and levofloxacin $(16 \mathrm{mg} / \mathrm{l})$ had much higher $\mathrm{MIC}_{90}$ values.

\section{MSSA isolates}

Ozenoxacin showed excellent activity (range $\leq 0.001-1 \mathrm{mg} / \mathrm{l})$ against MSSA isolates $(\mathrm{n}=247)$, with a MIC $_{50}$ and $\mathrm{MIC}_{90}$ of $0.004 \mathrm{mg} / \mathrm{l}$. Only two isolates had a MIC for ozenoxacin above $0.25 \mathrm{mg} / \mathrm{l}$ (MIC of $\left.1 \mathrm{mg} / \mathrm{l}\right) .14 \mathrm{MSSA}$ isolates were levofloxacin nonsusceptible (levofloxacin $\mathrm{MIC}_{50}=4 \mathrm{mg} / \mathrm{l}$ and $\mathrm{MIC}_{90} \geq 16 \mathrm{mg} / \mathrm{l}$ ): the $\mathrm{MIC}_{50}$ and $\mathrm{MIC}_{90}$ values for ozenoxacin against levofloxacin nonsusceptible MSSA isolates were 0.12 and $1 \mathrm{mg} / \mathrm{l}$, respectively. Lower $\mathrm{MIC}_{50}$ and $\mathrm{MIC}_{90}$ values (both $0.004 \mathrm{mg} / \mathrm{l}$ ) were found for ozenoxacin against the 231 levofloxacinsusceptible MSSA isolates (levofloxacin $\mathrm{MIC}_{50}=0.25 \mathrm{mg} / \mathrm{l}$ and $\mathrm{MIC}_{90}=0.25 \mathrm{mg} / \mathrm{l}$; Table 1 ). Comparative MIC data showed that ozenoxacin $\left(\mathrm{MIC}_{90}=0.004 \mathrm{mg} / \mathrm{l}\right)$ was more active than all reference compounds against MSSA isolates. The next most active compound was clindamycin $\left(\mathrm{MIC}_{90}=0.12 \mathrm{mg} / \mathrm{l}\right)$ which was 30-fold less active than ozenoxacin; followed by fusidic acid, mupirocin and retapamulin $\left(\mathrm{MIC}_{90}=0.25 \mathrm{mg} / \mathrm{l}\right)$; daptomycin, gentamicin, levofloxacin $\left(\mathrm{MIC}_{90}=0.5 \mathrm{mg} / \mathrm{l}\right)$; ciprofloxacin, amoxicillin-clavulanate, tetracycline, vancomycin $\left(\mathrm{MIC}_{90}=1 \mathrm{mg} / \mathrm{l}\right)$; cefuroxime and linezolid $\left(\mathrm{MIC}_{90}=2 \mathrm{mg} / \mathrm{l}\right)$; neomycin and ceftriaxone $\left(\mathrm{MIC}_{90}=4 \mathrm{mg} / \mathrm{l}\right)$; penicillin $\left(\mathrm{MIC}_{90}=16 \mathrm{mg} / \mathrm{l}\right)$; and erythromycin $\left(\mathrm{MIC}_{90}=64 \mathrm{mg} / \mathrm{l}\right.$; Table 1$)$.

\section{MRSA isolates}

Ozenoxacin was highly active against MRSA isolates $\left(\mathrm{n}=239\right.$ ), with a $\mathrm{MIC}_{50}$ of $0.12 \mathrm{mg} / \mathrm{l}$ and a MIC 90 of $0.25 \mathrm{mg} / \mathrm{l}$. One isolate had an ozenoxacin MIC of $\geq 4 \mathrm{mg} / \mathrm{l}$, and five isolates from four different sites had an ozenoxacin MIC of $2 \mathrm{mg} / \mathrm{l}$. Eight strains of MRSA from seven sites had an ozenoxacin MIC of $1 \mathrm{mg} / \mathrm{l}$. Ozenoxacin 


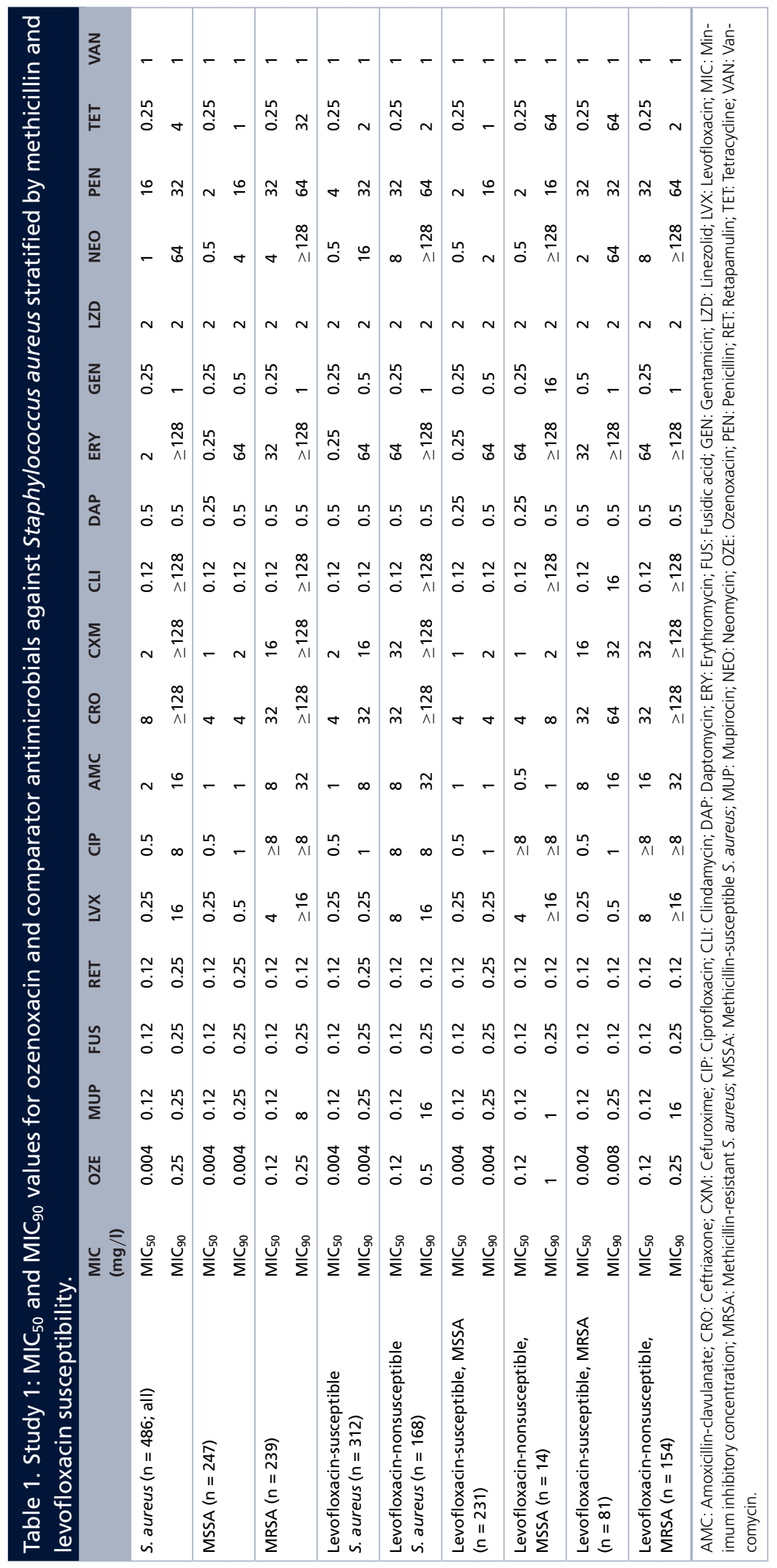


had a $\mathrm{MIC}_{50}$ of $0.004 \mathrm{mg} / \mathrm{l}$ and a $\mathrm{MIC}_{90}$ of $0.008 \mathrm{mg} / \mathrm{l}$ for levofloxacin-susceptible MRSA isolates $(\mathrm{n}=81$; levofloxacin $\mathrm{MIC}_{50}=0.25 \mathrm{mg} / \mathrm{l}$ and $\mathrm{MIC}_{90}=0.5 \mathrm{mg} / \mathrm{l}$ ). A total of $154 \mathrm{MRSA}$ isolates were also levofloxacinnonsusceptible (levofloxacin $\mathrm{MIC}_{50}=8 \mathrm{mg} / \mathrm{l}$ and $\mathrm{MIC}_{90} \geq 16 \mathrm{mg} / \mathrm{l}$ ). Ozenoxacin had a $\mathrm{MIC}_{50}$ and a $\mathrm{MIC}_{90}$ of 0.12 and $0.25 \mathrm{mg} / \mathrm{l}$, respectively, against levofloxacin-nonsusceptible MRSA isolates (Table 1). Comparative $\mathrm{MIC}_{90}$ data for all MRSA isolates tested showed that retapamulin $\left(\mathrm{MIC}_{90}=0.12 \mathrm{mg} / \mathrm{l}\right)$ was the most active compound, followed by ozenoxacin and fusidic acid $\left(\mathrm{MIC}_{90}=0.25 \mathrm{mg} / \mathrm{l}\right)$; daptomycin $\left(\mathrm{MIC}_{90}=0.5 \mathrm{mg} / \mathrm{l}\right)$; gentamicin and vancomycin $\left(\mathrm{MIC}_{90}=1 \mathrm{mg} / \mathrm{l}\right)$; linezolid $\left(\mathrm{MIC}_{90}=2 \mathrm{mg} / \mathrm{l}\right)$; mupirocin $\left(\mathrm{MIC}_{90}=8 \mathrm{mg} / \mathrm{l}\right)$; ciprofloxacin $\left(\mathrm{MIC}_{90} \geq 8 \mathrm{mg} / \mathrm{l}\right) ;$ levofloxacin $\left(\mathrm{MIC}_{90} \geq 16 \mathrm{mg} / \mathrm{l}\right) ;$ amoxicillin-clavulanate and tetracycline $\left(\mathrm{MIC}_{90}=32 \mathrm{mg} / \mathrm{l}\right)$; penicillin $\left(\mathrm{MIC}_{90}=64 \mathrm{mg} / \mathrm{l}\right)$; and ceftriaxone, cefuroxime, clindamycin, erythromycin and neomycin $\left(\mathrm{MIC}_{90}\right.$ $\geq 128 \mathrm{mg} / \mathrm{l}$; Table 1). Ozenoxacin was significantly more potent $\left(\mathrm{MIC}_{90}=0.008 \mathrm{mg} / \mathrm{l}\right)$ than all comparative compounds for levofloxacin-susceptible MRSA isolates. It was 15-fold more active than retapamulin and fusidic acid $\left(\mathrm{MIC}_{90}=0.12 \mathrm{mg} / \mathrm{l}\right)$, with the rank order of other compounds being: mupirocin $\left(\mathrm{MIC}_{90}=0.25 \mathrm{mg} / \mathrm{l}\right)$; daptomycin and levofloxacin $\left(\mathrm{MIC}_{90} 0.5 \mathrm{mg} / \mathrm{l}\right)$; ciprofloxacin; gentamicin and vancomycin $\left(\mathrm{MIC}_{90}=1 \mathrm{mg} / \mathrm{l}\right)$; linezolid $\left(\mathrm{MIC}_{90}=2 \mathrm{mg} / \mathrm{l}\right)$; clindamycin and amoxicillin-clavulanate $\left(\mathrm{MIC}_{90}=16 \mathrm{mg} / \mathrm{l}\right)$; cefuroxime and penicillin $\left(\mathrm{MIC}_{90}=32 \mathrm{mg} / \mathrm{l}\right)$; ceftriaxone, neomycin and tetracycline $\left(\mathrm{MIC}_{90}=64 \mathrm{mg} / \mathrm{l}\right)$; and erythromycin $\left(\mathrm{MIC}_{90}\right.$ $\geq 128 \mathrm{mg} / \mathrm{l}$; Table 1). Against MRSA isolates $(\mathrm{n}=239)$, including those which were levofloxacin susceptible $(\mathrm{n}=81)$ and nonsusceptible $(\mathrm{n}=154)$, ozenoxacin $\left(\mathrm{MIC}_{90}=0.25 \mathrm{ug} / \mathrm{ml}\right)$ was 32 -fold more active than mupirocin $\left(\mathrm{MIC}_{90}=8 \mu \mathrm{g} / \mathrm{ml}\right.$; Table 1$)$.

\section{Study 2}

The antibacterial activity of ozenoxacin and comparators against $S$. aureus, stratified by methicillin and levofloxacin susceptibility, is summarized in Table 2. Of the 504 S. aureus isolates included in the study, 225 (44.6\%) were MRSA and $279(55.4 \%)$ were MSSA. Almost half (45.7\%) of the MRSA isolates were also nonsusceptible to levofloxacin, whereas only $6.5 \%$ of MSSA isolates were levofloxacin nonsusceptible. Ozenoxacin demonstrated excellent overall activity $\left(\mathrm{MIC}_{50}=0.002 \mathrm{mg} / \mathrm{l}, \mathrm{MIC}_{90}=0.06 \mathrm{mg} / \mathrm{l}\right)$ against all $504 \mathrm{~S}$. aureus isolates, inhibiting $99.4 \%$ at a MIC of $\leq 0.5 \mathrm{mg} / \mathrm{l}$. The activity of ozenoxacin was higher against levofloxacin-susceptible $S$. aureus isolates $\left(\mathrm{MIC}_{50}\right.$ and $\left.\mathrm{MIC}_{90}=0.002 \mathrm{mg} / \mathrm{l}\right)$ compared with levofloxacin nonsusceptible isolates $\left(\mathrm{MIC}_{50}=0.06 \mathrm{mg} / \mathrm{l} ; \mathrm{MIC}_{90}=0.5 \mathrm{mg} / \mathrm{l}\right.$; Table 2). MIC values to ozenoxacin in MRSA isolates $\left(\mathrm{MIC}_{50}=0.004 \mathrm{mg} / \mathrm{l} ; \mathrm{MIC}_{90}=0.12 \mathrm{mg} / \mathrm{l}\right)$ were slightly higher than those found with all $S$. aureus isolates.

Ozenoxacin was the most potent agent against all $S$. aureus isolates tested. Comparing $\mathrm{MIC}_{50}$ values, ozenoxacin $\left(\mathrm{MIC}_{50}=0.002 \mathrm{mg} / \mathrm{l}\right)$ had 32-fold greater activity than clindamycin $\left(\mathrm{MIC}_{50}=0.06 \mathrm{mg} / \mathrm{l}\right) ; 64$-fold greater activity than retapamulin or fusidic acid $\left(\mathrm{MIC}_{50}=0.12 \mathrm{mg} / \mathrm{l}\right)$; and 128-fold greater activity than erythromycin, mupirocin, ciprofloxacin or levofloxacin $\left(\mathrm{MIC}_{50}=0.25 \mathrm{mg} / \mathrm{l}\right)$. At the $\mathrm{MIC}_{90}$ level, ozenoxacin $\left(\mathrm{MIC}_{90}=0.06 \mathrm{mg} / \mathrm{l}\right.$ ) was twofold more potent than retapamulin $\left(\mathrm{MIC}_{90}=0.12 \mathrm{mg} / \mathrm{l}\right)$; fourfold more potent than fusidic acid $\left(\mathrm{MIC}_{90}=0.25 \mathrm{mg} / \mathrm{l}\right)$; eightfold more active than mupirocin $\left(\mathrm{MIC}_{90}=0.5 \mathrm{mg} / \mathrm{l}\right)$; and at least 256 -fold more potent than erythromycin, clindamycin, ciprofloxacin or levofloxacin $\left(\mathrm{MIC}_{90} \geq 16 \mathrm{mg} / 1\right.$; Table 2).

Against MRSA, ozenoxacin $\left(\mathrm{MIC}_{50}=0.004 \mathrm{mg} / \mathrm{l} ; \mathrm{MIC}_{90}=0.12 \mathrm{mg} / \mathrm{l}\right)$ had greater activity than mupirocin $\left(\mathrm{MIC}_{50}=0.25 \mathrm{mg} / \mathrm{l} ; \mathrm{MIC}_{90}=0.5 \mathrm{mg} / \mathrm{l}\right)$ and fusidic acid $\left(\mathrm{MIC}_{50}=0.12 \mathrm{mg} / \mathrm{l} ; \mathrm{MIC}_{90}=0.25 \mathrm{mg} / \mathrm{l}\right)$, and using $\mathrm{MIC}_{50}$ values, was more potent than retapamulin $\left(\mathrm{MIC}_{50}=0.12 \mathrm{mg} / \mathrm{l} ; \mathrm{MIC}_{90}=0.12 \mathrm{mg} / \mathrm{l}\right)$. Against levofloxacin nonsusceptible $S$. aureus, ozenoxacin $\left(\mathrm{MIC}_{50}=0.06 \mathrm{mg} / \mathrm{l} ; \mathrm{MIC}_{90}=0.5 \mathrm{mg} / \mathrm{l}\right)$ had greater activity than mupirocin $\left(\mathrm{MIC}_{50}=0.25 \mathrm{mg} / \mathrm{l} ; \mathrm{MIC}_{90}=2 \mathrm{mg} / \mathrm{l}\right)$ and fusidic acid $\left(\mathrm{MIC}_{50}=0.12 \mathrm{mg} / \mathrm{l} ; \mathrm{MIC}_{90}=0.25 \mathrm{mg} / \mathrm{l}\right)$, and was similar to retapamulin using $\mathrm{MIC}_{50}$ values (Table 2). Whereas $\mathrm{MIC}_{50}$ values for clindamycin and ozenoxacin were both $0.06 \mathrm{mg} / \mathrm{l}, \mathrm{MIC}_{90}$ values indicated that ozenoxacin $\left(\mathrm{MIC}_{90}=0.5 \mathrm{mg} / \mathrm{l}\right)$ was at least 64 -fold more potent than clindamycin $\left(\mathrm{MIC}_{90}>16 \mathrm{mg} / \mathrm{l}\right)$. The remaining agents had higher $\mathrm{MIC}_{50}$ and $\mathrm{MIC}_{90}$ values than ozenoxacin (Table 2).

Table 3 shows the proportion of $S$. aureus, MSSA, MRSA and levofloxacin susceptible or resistant $S$. aureus isolates, which were susceptible, resistant or had intermediate status to 11 antimicrobials, as assessed using EUCAST and CLSI criteria. Up to approximately $7 \%$ of $S$. aureus, MRSA, and levofloxacin susceptible or resistant $S$. aureus isolates were resistant to mupirocin and fusidic acid. 


$$
\mid
$$




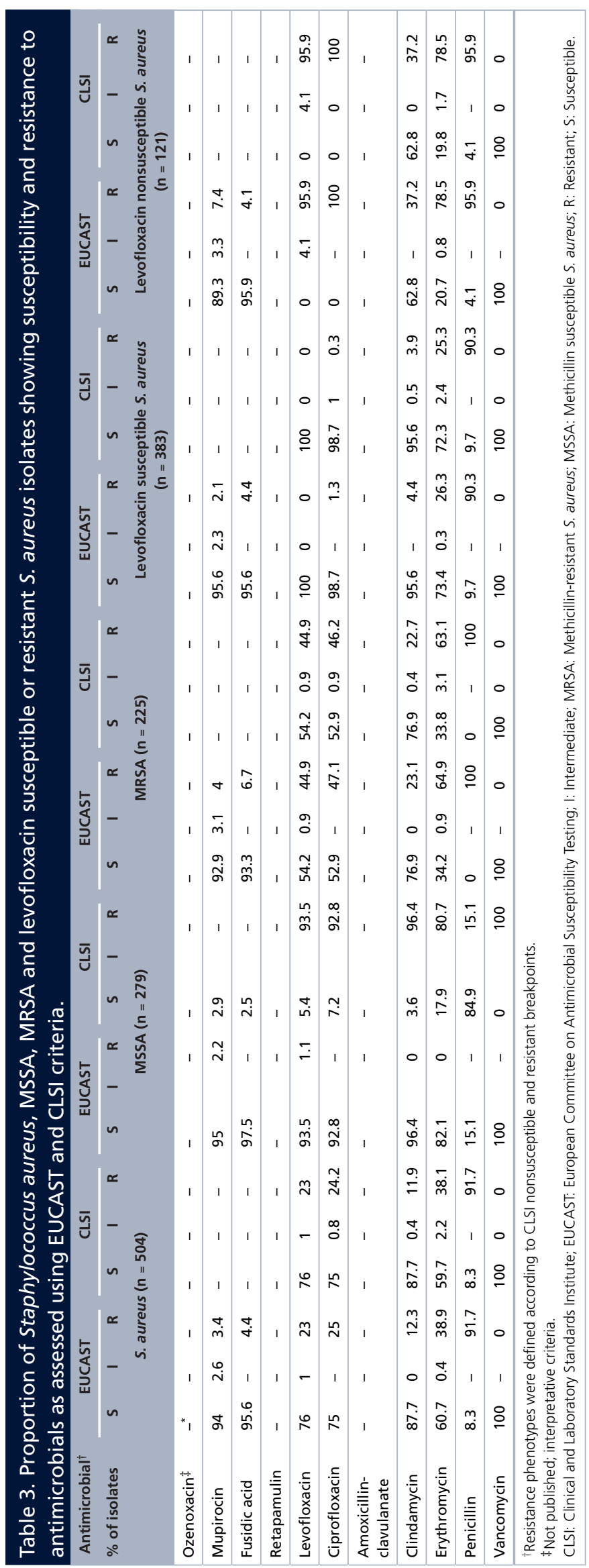


Streptococcus species

S. pyogenes study 1

Ozenoxacin was highly active (range: $0.008-0.06 \mathrm{mg} / \mathrm{l}$ ) against 217 S. pyogenes isolates, with a MIC 50 of $0.03 \mathrm{mg} / 1$ and $\mathrm{MIC}_{90}$ of $0.06 \mathrm{mg} / \mathrm{l}$. The most active compounds against $S$. pyogenes were cefuroxime $\left(\mathrm{MIC}_{90} \leq 0.015 \mathrm{mg} / \mathrm{l}\right)$; penicillin $\left(\mathrm{MIC}_{90}=0.015 \mathrm{mg} / \mathrm{l}\right)$; ceftriaxone and amoxicillin-clavulanate $\left(\mathrm{MIC}_{90}=0.03 \mathrm{mg} / \mathrm{l}\right)$; ozenoxacin, clindamycin, daptomycin, erythromycin and retapamulin $\left(\mathrm{MIC}_{90}=0.06 \mathrm{mg} / \mathrm{l}\right)$; and mupirocin $\left(\mathrm{MIC}_{90}=0.12 \mathrm{mg} / \mathrm{l}\right)$. S. pyogenes isolates were less susceptible to ciprofloxacin and vancomycin $\left(\mathrm{MIC}_{90}=0.5 \mathrm{mg} / \mathrm{l}\right)$; levofloxacin and linezolid $\left(\mathrm{MIC}_{90}=1 \mathrm{mg} / \mathrm{l}\right) ;$ fusidic acid and gentamicin $\left(\mathrm{MIC}_{90}=8 \mathrm{mg} / \mathrm{l}\right)$; tetracycline $\left(\mathrm{MIC}_{90}=32 \mathrm{mg} / \mathrm{l}\right) ;$ and neomycin $\left(\mathrm{MIC}_{90}=64 \mathrm{mg} / \mathrm{l}\right.$; Table 4). Thus, against $S$. pyogenes isolates ozenoxacin was equipotent to retapamulin, had twofold higher activity than mupirocin and 13-fold higher activity than fusidic acid.

\section{S. agalactiae study 1}

Ozenoxacin was highly active (range: $0.008-0.06 \mathrm{mg} / \mathrm{l}$ ) against the $37 \mathrm{~S}$. agalactiae isolates tested, with a MIC 50 of $0.03 \mathrm{mg} / \mathrm{l}$ and $\mathrm{MIC}_{90}$ of $0.06 \mathrm{mg} / \mathrm{l}$. Ozenoxacin, together with penicillin and retapamulin $\left(\mathrm{MIC}_{90}=0.06 \mathrm{mg} / \mathrm{l}\right)$, were the most active compounds against $S$. agalactiae. The rank order of the remaining compounds was: ceftriaxone, cefuroxime and amoxicillin-clavulanate $\left(\mathrm{MIC}_{90}=0.12 \mathrm{mg} / \mathrm{l}\right)$; daptomycin $\left(\mathrm{MIC}_{90}=0.25 \mathrm{mg} / \mathrm{l}\right)$; vancomycin $\left(\mathrm{MIC}_{90}=0.5 \mathrm{mg} / \mathrm{l}\right)$; ciprofloxacin, levofloxacin and mupirocin $\left(\mathrm{MIC}_{90}=1 \mathrm{mg} / \mathrm{l}\right) ;$ linezolid $(\mathrm{MIC} 90=2 \mathrm{mg} / \mathrm{l})$; fusidic acid $\left(\mathrm{MIC}_{90}=16 \mathrm{mg} / \mathrm{l}\right)$; gentamicin $\left(\mathrm{MIC}_{90}=32 \mathrm{mg} / \mathrm{l}\right)$; tetracycline $\left(\mathrm{MIC}_{90}=64 \mathrm{mg} / \mathrm{l}\right)$; clindamycin and erythromycin $\left(\mathrm{MIC}_{90} \geq 64 \mathrm{mg} / \mathrm{l}\right)$; and neomycin $\left(\mathrm{MIC}_{90} \geq 128 \mathrm{mg} / \mathrm{l}\right.$; Table 4$)$.

\section{S. pneumoniae study 1}

Ozenoxacin was highly active (range: $0.015-0.06 \mathrm{mg} / \mathrm{l}$ ) against $S$. pneumoniae isolates $(\mathrm{n}=29)$, with a $\mathrm{MIC}_{50}$ and $\mathrm{MIC}_{90}$ of 0.03 and $0.06 \mathrm{mg} / 1$, respectively. In comparison to the reference compounds, ozenoxacin $\left(\mathrm{MIC}_{90}=0.06 \mathrm{mg} / \mathrm{l}\right)$ was significantly the most active. The rank order of the reference compounds was: daptomycin $\left(\mathrm{MIC}_{90}=0.25 \mathrm{mg} / \mathrm{l}\right)$; retapamulin and vancomycin $\left(\mathrm{MIC}_{90}=0.5 \mathrm{mg} / \mathrm{l}\right) ;$ levofloxacin $(\mathrm{MIC} 90=1 \mathrm{mg} / \mathrm{l})$; ceftriaxone, ciprofloxacin, linezolid and mupirocin $\left(\mathrm{MIC}_{90}=2 \mathrm{mg} / \mathrm{l}\right)$; penicillin $\left(\mathrm{MIC}_{90}=4 \mathrm{mg} / \mathrm{l}\right)$; amoxicillinclavulanate and gentamicin $\left(\mathrm{MIC}_{90}=8 \mathrm{mg} / \mathrm{l}\right)$; cefuroxime $\left(\mathrm{MIC}_{90}=16 \mathrm{mg} / \mathrm{l}\right)$; fusidic acid and tetracycline $\left(\mathrm{MIC}_{90}=32 \mathrm{mg} / \mathrm{l}\right) ;$ neomycin $\left(\mathrm{MIC}_{90}=64 \mathrm{mg} / \mathrm{l}\right) ;$ and clindamycin and erythromycin $(\mathrm{MIC} 90 \geq 64 \mathrm{mg} / \mathrm{l}$; Table 4).

\section{S. pyogenes \& $S$. agalactiae study 2}

The antibacterial activity of ozenoxacin and comparators against $S$. pyogenes $(\mathrm{n}=124)$ and $S$. agalactiae $(\mathrm{n}=88)$ is summarized in Table 4. All isolates were susceptible to penicillin (MIC $\leq 0.12 \mathrm{mg} / \mathrm{l}$ ), amoxicillin-clavulanate (MIC $\leq 4 / 2 \mathrm{mg} / \mathrm{l})$ and vancomycin (MIC $\leq 0.5 \mathrm{mg} / \mathrm{l})$. All $S$. pyogenes isolates and $95.5 \%(84 / 88)$ of $S$. agalactiae isolates were susceptible to levofloxacin by CLSI interpretative criteria (Table 4). Ozenoxacin was highly active against both S. pyogenes and $S$. agalactiae. $\mathrm{MIC}_{50}$ and $\mathrm{MIC}_{90}$ values were 0.008 and $0.015 \mathrm{mg} / 1$, respectively, against $S$. pyogenes and were 0.015 and $0.03 \mathrm{mg} / \mathrm{l}$, respectively, against $S$. agalactiae (Table 4).

Ozenoxacin was the most potent $\left(\mathrm{MIC}_{50}=0.008 \mathrm{mg} / \mathrm{l} ; \mathrm{MIC}_{90}=0.015 \mathrm{mg} / \mathrm{l}\right)$ agent tested against all S. pyogenes isolates, inhibiting $98.3 \%$ at a $\mathrm{MIC}$ of $\leq 0.03 \mathrm{mg} / \mathrm{l}$. Ozenoxacin was fourfold more active than erythromycin, clindamycin or retapamulin $\left(\mathrm{MIC}_{50}=0.03 \mathrm{mg} / 1 ; \mathrm{MIC}_{90}=0.06 \mathrm{mg} / \mathrm{l}\right)$, at least eightfold more active than mupirocin $\left(\mathrm{MIC}_{50}=0.06 \mathrm{mg} / \mathrm{l} ; \mathrm{MIC}_{90}=0.25 \mathrm{mg} / \mathrm{l}\right), 64$-fold more active than ciprofloxacin or levofloxacin $\left(\mathrm{MIC}{ }_{50}=0.5 \mathrm{mg} / \mathrm{l}\right.$; $\left.\mathrm{MIC}_{90}=1 \mathrm{mg} / \mathrm{l}\right)$ and at least 256-fold more active than fusidic acid $\left(\mathrm{MIC}_{50}\right.$ and $\mathrm{MIC}_{90}=4 \mathrm{mg} / \mathrm{l}$; Table 4).

Ozenoxacin was also the most potent $\left(\mathrm{MIC}_{50}=0.015 \mathrm{mg} / 1 ; \mathrm{MIC}_{90}=0.03 \mathrm{mg} / \mathrm{l}\right)$ agent tested against $S$. agalactiae isolates, inhibiting $95.5 \%$ of isolates at a MIC of $\leq 0.03 \mathrm{mg} / \mathrm{l}$. Ozenoxacin was at least twofold more active than erythromycin or clindamycin $\left(\mathrm{MIC}_{50}=0.03 \mathrm{mg} / \mathrm{l} ; \mathrm{MIC}_{90}>16 \mathrm{mg} / \mathrm{l}\right)$; fourfold more active than retapamulin $\left(\mathrm{MIC}_{50}=0.06 \mathrm{mg} / \mathrm{l} ; \mathrm{MIC}_{90}=0.12 \mathrm{mg} / \mathrm{l}\right) ; 32$-fold more active than ciprofloxacin or levofloxacin $\left(\mathrm{MIC} \mathrm{C}_{50}=\mathrm{mg} / \mathrm{l} ;\right.$ $\left.\mathrm{MIC}_{90}=0.5 / 1 \mathrm{mg} / \mathrm{l}\right)$; at least 32-fold more active than mupirocin $\left(\mathrm{MIC}_{50}\right.$ and $\left.\mathrm{MIC}_{90}=1 \mathrm{mg} / \mathrm{l}\right)$; and 512 -fold more active than fusidic acid $\left(\mathrm{MIC}_{50}=8 \mathrm{mg} / \mathrm{l} ; \mathrm{MIC}_{90}=16 \mathrm{mg} / \mathrm{l}\right.$; Table 4).

\section{S. epidermidis study 1}

$\mathrm{MIC}_{50}$ and $\mathrm{MIC}_{90}$ values for ozenoxacin and comparator antimicrobial agents against $S$. epidermidis isolates, which were also stratified by methicillin and levofloxacin susceptibility, are shown in Table 5. Ozenoxacin was highly active against $S$. epidermidis isolates $(\mathrm{n}=190)$, with a $\mathrm{MIC}_{50}$ of $0.06 \mathrm{mg} / \mathrm{l}$ and a $\mathrm{MIC}_{90}$ of $1 \mathrm{mg} / \mathrm{l}$. Two isolates from different sites had an ozenoxacin MIC of $\geq 4 \mathrm{mg} / \mathrm{l}$ and 12 isolates from nine sites had an ozenoxacin MIC of $2 \mathrm{mg} / 1$. 


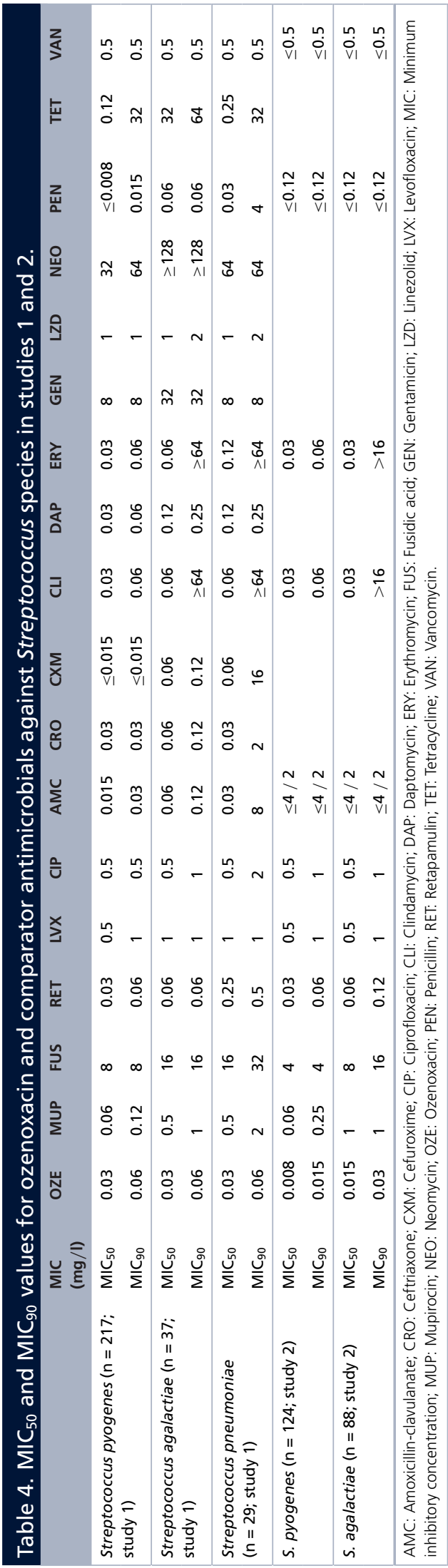




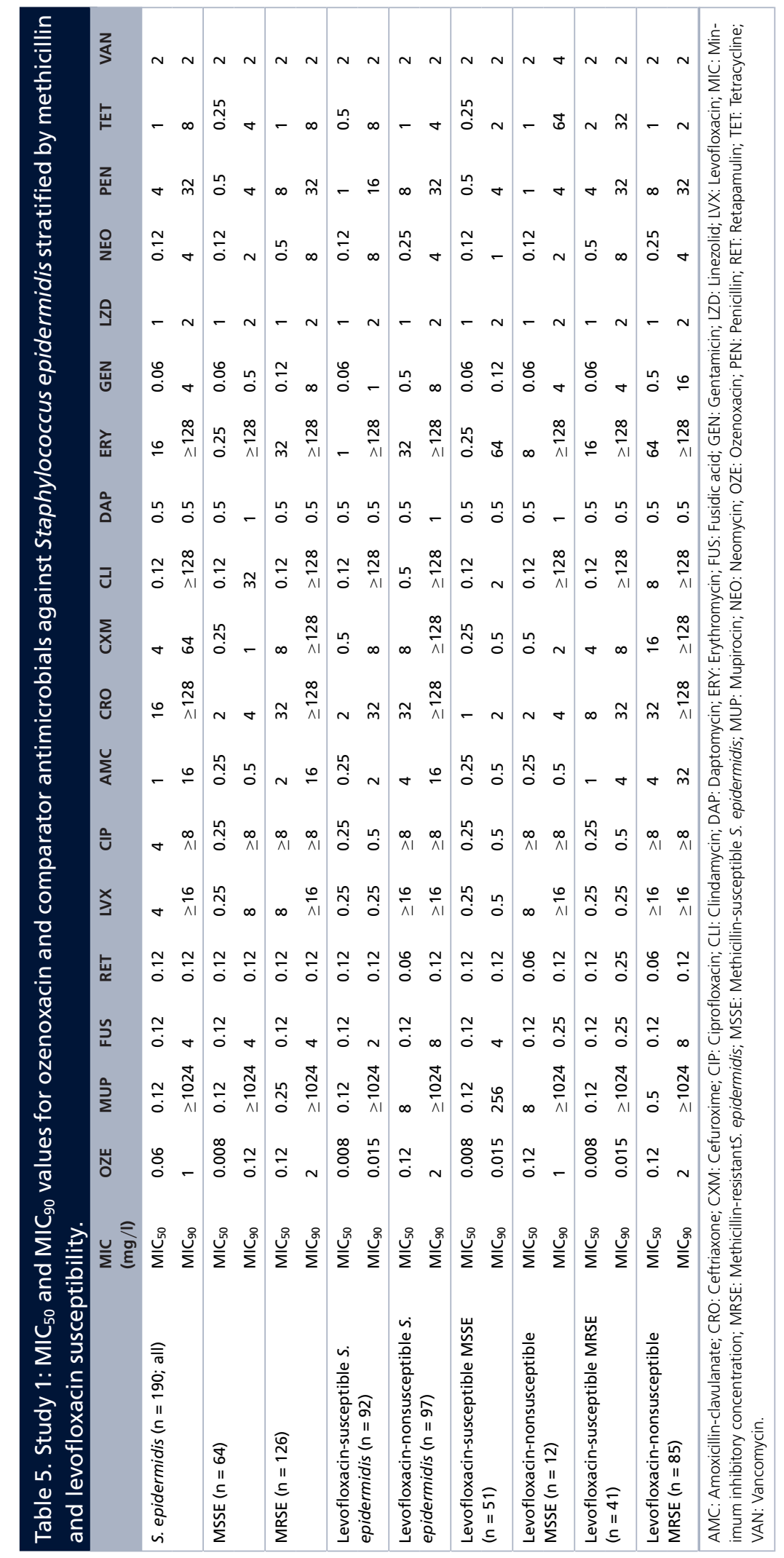




\begin{tabular}{|c|c|c|c|c|c|c|c|c|c|c|c|c|}
\hline & $\begin{array}{l}\text { MIC } \\
(\mathrm{mg} / \mathrm{l})\end{array}$ & OZE & MUP & FUS & RET & LVX & CIP & AMC & CLI & ERY & PEN & VAN \\
\hline \multirow[t]{2}{*}{ S. epidermidis ( $n=195 ;$ all) } & $\mathrm{MIC}_{50}$ & 0.008 & 0.25 & 0.12 & 0.06 & 0.25 & 0.5 & $\leq 4 / 2$ & 0.06 & $>16$ & $>0.25$ & 1 \\
\hline & $\mathrm{MIC}_{90}$ & 0.25 & $>256$ & 8 & 0.25 & $>16$ & $>16$ & $>8 / 4$ & $>16$ & $>16$ & $>0.25$ & 2 \\
\hline \multirow[t]{2}{*}{ MSSE $(n=86)$} & $\mathrm{MIC}_{50}$ & 0.004 & 0.25 & 0.12 & 0.06 & 0.25 & 0.25 & $\leq 4 / 2$ & 0.06 & $>16$ & $>0.25$ & 1 \\
\hline & $\mathrm{MIC}_{90}$ & 0.03 & 256 & 8 & 0.25 & 4 & 4 & $\leq 4 / 2$ & 0.12 & $>16$ & $>0.25$ & 2 \\
\hline \multirow[t]{2}{*}{ MRSE $(n=109)$} & $\mathrm{MIC}_{50}$ & 0.06 & 0.25 & 0.12 & 0.06 & 4 & 8 & $\leq 4 / 2$ & 0.25 & $>16$ & $>0.25$ & 1 \\
\hline & $\mathrm{MIC}_{90}$ & 0.5 & $>256$ & 16 & 0.12 & $>16$ & $>16$ & $>8 / 4$ & $>16$ & $>16$ & $>0.25$ & 2 \\
\hline \multirow{2}{*}{$\begin{array}{l}\text { Levofloxacin susceptible } S \text {. } \\
\text { epidermidis }(n=105)\end{array}$} & $\mathrm{MIC}_{50}$ & 0.004 & 0.25 & 0.12 & 0.06 & 0.25 & 0.25 & $\leq 4 / 2$ & 0.06 & $>16$ & $>0.25$ & 1 \\
\hline & $\mathrm{MIC}_{90}$ & 0.008 & $>256$ & 4 & 0.25 & 0.25 & 0.5 & $\leq 4 / 2$ & 0.12 & $>16$ & $>0.25$ & 2 \\
\hline \multirow{2}{*}{$\begin{array}{l}\text { Levofloxacin nonsusceptible } \\
\text { S. epidermidis }(n=90)\end{array}$} & $\mathrm{MIC}_{50}$ & 0.06 & 0.25 & 0.12 & 0.06 & 8 & $>16$ & $\leq 4 / 2$ & $>16$ & $>16$ & $>0.25$ & 1 \\
\hline & $\mathrm{MIC}_{90}$ & 1 & $>256$ & 16 & 0.12 & $>16$ & $>16$ & $>8 / 4$ & $>16$ & $>16$ & $>0.25$ & 2 \\
\hline
\end{tabular}

13 strains of S. epidermidis from ten sites had an ozenoxacin MIC of $1 \mathrm{mg} / \mathrm{l}$ (Table 5). Against all S. epidermidis isolates tested $(\mathrm{n}=190)$, retapamulin $\left(\mathrm{MIC}_{90}=0.12 \mathrm{mg} / \mathrm{l}\right)$ was the most active compound. Ozenoxacin $\left(\mathrm{MIC}_{90}=\right.$ $1 \mathrm{mg} / \mathrm{l})$ was fourfold more active than fusidic acid $\left(\mathrm{MIC}_{90}=4 \mathrm{mg} / \mathrm{l}\right)$ and more than 1000 -fold more active than mupirocin $\left(\mathrm{MIC}_{90} \geq 1024 \mathrm{mg} / \mathrm{l}\right)$. Daptomycin $\left(\mathrm{MIC}_{90}=0.5 \mathrm{mg} / \mathrm{l}\right)$ was the second most active compound tested (Table 5).

Ozenoxacin was highly active against methicillin-susceptible $S$. epidermidis (MSSE) isolates ( $\mathrm{n}=64)$ with a $\mathrm{MIC}_{50}$ of $0.008 \mathrm{mg} / \mathrm{l}$ and a $\mathrm{MIC}_{90}$ of $0.12 \mathrm{mg} / \mathrm{l} .12 \mathrm{MSSE}$ isolates were levofloxacin nonsusceptible (levofloxacin $\mathrm{MIC}_{50}=8 \mathrm{mg} / \mathrm{l}$ and $\mathrm{MIC}_{90} \geq 16 \mathrm{mg} / \mathrm{l}$ ): the ozenoxacin $\mathrm{MIC}_{50}$ and $\mathrm{MIC}_{90}$ were 0.12 and $1 \mathrm{mg} / \mathrm{l}$, respectively. An ozenoxacin $\mathrm{MIC}_{50}$ of $0.008 \mathrm{mg} / \mathrm{l}$ and a $\mathrm{MIC}_{90}$ of $0.015 \mathrm{mg} / \mathrm{l}$ were found for levofloxacin-susceptible MSSE isolates ( $\mathrm{n}=51$; levofloxacin $\mathrm{MIC}_{50}=0.25 \mathrm{mg} / \mathrm{l}$ and $\mathrm{MIC}_{90}=0.5 \mathrm{mg} / \mathrm{l}$; Table 5).

Ozenoxacin was highly active against methicillin-resistant $S$. epidermidis (MRSE) isolates $(\mathrm{n}=126)$, with a $\mathrm{MIC}_{50}$ of $0.12 \mathrm{mg} / \mathrm{l}$ and a $\mathrm{MIC}_{90}$ of $2 \mathrm{mg} / \mathrm{l}$. For levofloxacin nonsusceptible S. epidermidis isolates $(\mathrm{n}=97$; levofloxacin $\mathrm{MIC}_{50} \geq 16 \mathrm{mg} / \mathrm{l}$ and $\mathrm{MIC}_{90} \geq 16 \mathrm{mg} / \mathrm{l}$ ) the ozenoxacin $\mathrm{MIC}_{50}$ was $0.12 \mathrm{mg} / \mathrm{l}$ and the $\mathrm{MIC}_{90}$ was $2 \mathrm{mg} /$ l. Ozenoxacin was much more active against levofloxacin-susceptible $S$. epidermidis isolates $(\mathrm{n}=92$; levofloxacin $\mathrm{MIC}_{50}=0.25 \mathrm{mg} / \mathrm{l}$ and $\mathrm{MIC}_{90}=0.25 \mathrm{mg} / \mathrm{l}$ ), with $\mathrm{MIC}_{50}$ and $\mathrm{MIC}_{90}$ values of 0.008 and $0.015 \mathrm{mg} / \mathrm{l}$, respectively (Table 5). Against levofloxacin susceptible S. epidermidis $(\mathrm{n}=92)$, including MSSE $(\mathrm{n}=51)$ and MRSE $(\mathrm{n}=41)$, ozenoxacin ( $\mathrm{MIC}_{90}=0.015 \mathrm{mg} / \mathrm{l}$ against all isolates) was at least eightfold more active than retapamulin $\left(\mathrm{MIC}_{90}=0.12,0.12\right.$ and $0.25 \mathrm{mg} / \mathrm{l}$, respectively $)$ and at least 128 -fold more active than fusidic acid $\left(\mathrm{MIC}_{90}=2,4\right.$ and $0.25 \mathrm{mg} / \mathrm{l}$, respectively). The respective $\mathrm{MIC}_{90}$ values of mupirocin against this group of isolates were $\geq 1024$, 256 and $\geq 1024 \mathrm{mg} / \mathrm{l}$ (Table 5).

\section{S. epidermidis study 2}

The antibacterial activity of ozenoxacin and comparators against $S$. epidermidis, stratified by methicillin and levofloxacin susceptibility, is shown in Table 6. Of the 195 S. epidermidis isolates included in the study, 109 $(55.9 \%)$ were methicillin-resistant and $86(44.1 \%)$ were methicillin-susceptible. Levofloxacin resistance among MRSE and MSSE isolates was 72.5 and $12.8 \%$, respectively (Table 6). Ozenoxacin was the most potent agent tested against $S$. epidermidis $\left(\mathrm{MIC}_{50}=0.008 ; \mathrm{MIC}_{90}=0.25 \mathrm{mg} / \mathrm{l}\right)$, in common with data for all coagulase-negative staphylococci isolates. The activity of ozenoxacin was higher against MSSE isolates $\left(n=86 ; M_{50}=0.004\right.$; $\left.\mathrm{MIC}_{90}=0.03\right)$ than against MRSE isolates $\left(\mathrm{n}=109 ; \mathrm{MIC}_{50}=0.06 ; \mathrm{MIC}_{90}=0.5\right)$. The activity of ozenoxacin was also higher against levofloxacin-susceptible $S$. epidermidis isolates $\left(\mathrm{n}=105 ; \mathrm{MIC}_{50}=0.004 ; \mathrm{MIC}_{90}=0.008\right)$ than against levofloxacin nonsusceptible $S$. epidermidis isolates $\left(\mathrm{n}=90 ; \mathrm{MIC}_{50}=0.06 ; \mathrm{MIC}_{90}=1\right)$ regardless of the methicillin resistance status (Table 6).

Comparative analyses of $\mathrm{MIC}_{50}$ values against $S$. epidermidis showed that ozenoxacin $\left(\mathrm{MIC}_{50}=0.008 \mathrm{mg} / \mathrm{l}\right) \mathrm{had}$ eightfold greater activity than clindamycin and retapamulin $\left(\mathrm{MIC}_{50}=0.06 \mathrm{mg} / \mathrm{l}\right) ; 16$-fold greater activity than fusidic acid $\left(\mathrm{MIC}_{50}=0.12 \mathrm{mg} / \mathrm{l}\right) ; 32$-fold greater activity than mupirocin or levofloxacin $\left(\mathrm{MIC}_{50}=0.25 \mathrm{mg} / \mathrm{l}\right)$; 
64-fold greater activity than ciprofloxacin $\left(\mathrm{MIC}_{50}=0.5 \mathrm{mg} / \mathrm{l}\right)$; and at least greater than 256-fold greater activity than erythromycin ( $\mathrm{MIC}_{50}>16 \mathrm{mg} / \mathrm{l}$; Table 6).

Against levofloxacin nonsusceptible S. epidermidis isolates, retapamulin $\left(\mathrm{MIC}_{50}=0.06 \mathrm{mg} / \mathrm{l} ; \mathrm{MIC}_{90}=0.12 \mathrm{mg} / \mathrm{l}\right)$ was the most active antimicrobial agent tested, with ozenoxacin $\left(\mathrm{MIC}_{50}=0.06 \mathrm{mg} / \mathrm{l} ; \mathrm{MIC}_{90}=1 \mathrm{mg} / \mathrm{l}\right) \mathrm{ranked}$ second in potency of all tested compounds (Table 6).

Ozenoxacin had higher activity against MRSE $\left(\mathrm{MIC}_{50}=0.06 \mathrm{mg} / \mathrm{l} ; \mathrm{MIC}_{90}=0.5 \mathrm{mg} / \mathrm{l}\right)$ than mupirocin $\left(\mathrm{MIC}_{50}=0.25 \mathrm{mg} / \mathrm{l} ; \mathrm{MIC}_{90}>256 \mathrm{mg} / \mathrm{l}\right)$ and fusidic acid $\left(\mathrm{MIC}_{50}=0.12 \mathrm{mg} / \mathrm{l} ; \mathrm{MIC}_{90}=16 \mathrm{mg} / \mathrm{l}\right)$. Against levofloxacin nonsusceptible $S$. epidermidis, ozenoxacin $\left(\mathrm{MIC}_{50}=0.06 \mathrm{mg} / \mathrm{l} ; \mathrm{MIC}_{90}=1 \mathrm{mg} / \mathrm{l}\right)$ was more potent than mupirocin $\left(\mathrm{MIC}_{50}=0.25 \mathrm{mg} / \mathrm{l} ; \mathrm{MIC}_{90}>256 \mathrm{mg} / \mathrm{l}\right)$ and fusidic acid $\left(\mathrm{MIC}_{50}=0.12 \mathrm{mg} / \mathrm{l} ; \mathrm{MIC}_{90}=16 \mathrm{mg} / \mathrm{l}\right.$; Table 6).

\section{CNS species other than S. epidermidis: study 1}

For CNS spp. which were not strains of $S$. epidermidis ( $\mathrm{n}=37$ ), oxenoxacin was more active than all reference compounds with an $\mathrm{MIC}_{50}$ of $0.008 \mathrm{mg} / \mathrm{l}$ and a $\mathrm{MIC}_{90}$ of $0.12 \mathrm{mg} / \mathrm{l}$. In comparison, the rank order of reference compounds was daptomycin $\left(\mathrm{MIC}_{90}=0.5 \mathrm{mg} / \mathrm{l}\right)$; linezolid $\left(\mathrm{MIC}_{90}=1 \mathrm{mg} / \mathrm{l}\right)$; neomycin, retapamulin and vancomycin $\left(\mathrm{MIC}_{90}=2 \mathrm{mg} / \mathrm{l}\right)$; gentamicin and tetracycline $\left(\mathrm{MIC}_{90}=4 \mathrm{mg} / \mathrm{l}\right)$; ciprofloxacin, amoxicillin-clavulanate and levofloxacin $\left(\mathrm{MIC}_{90}=8 \mathrm{mg} / \mathrm{l}\right)$; fusidic acid and penicillin $\left(\mathrm{MIC}_{90}=16 \mathrm{mg} / \mathrm{l}\right)$; ceftriaxone and cefuroxime $\left(\mathrm{MIC}_{90}=64 \mathrm{mg} / \mathrm{l}\right)$; clindamycin and erythromycin $\left(\mathrm{MIC}_{90} \geq 128 \mathrm{mg} / \mathrm{l}\right)$; and mupirocin $\left(\mathrm{MIC}_{90} \geq 1024 \mathrm{mg} / \mathrm{l}\right.$; Table 7).

\section{CNS species other than S. epidermidis: study 2}

Table 7 summarizes the antibacterial activity of ozenoxacin and comparators against CNS, stratified by methicillin and levofloxacin susceptibility. Of the 315 CNS isolates included in the study, 146 (46.3\%) were methicillinresistant (MR-CNS) and 169 (53.7\%) were methicillin-susceptible (MS-CNS). Most of the MR-CNS isolates (72.6\%) were nonsusceptible to levofloxacin, compared with only $8.3 \%$ of the MS-CNS isolates. Ozenoxacin was highly active against all CNS isolates $\left(\mathrm{MIC}_{50}=0.008 \mathrm{mg} / \mathrm{l} ; \mathrm{MIC}_{90}=0.12 \mathrm{mg} / \mathrm{l}\right)$ inhibiting $96.8 \%$ of isolates at a MIC of $\leq 0.5 \mathrm{mg} / \mathrm{l}$. Ozenoxacin tested against levofloxacin-nonsusceptible CNS isolates $\left(\mathrm{MIC}_{50}=0.06 \mathrm{mg} / \mathrm{l}\right.$; $\mathrm{MIC}_{90}=0.5 \mathrm{mg} / \mathrm{l}$ ) showed MIC values at least 16-fold higher than isolates with levofloxacin susceptibility $\left(\mathrm{MIC}_{50}=0.004 \mathrm{mg} / \mathrm{l} ; \mathrm{MIC}_{90}=0.008 \mathrm{mg} / \mathrm{l}\right)$. Similar results were obtained for MR-CNS $\left(\mathrm{MIC}_{50}=0.06 \mathrm{mg} / \mathrm{l}\right.$; $\left.\mathrm{MIC}_{90}=0.5 \mathrm{mg} / \mathrm{l}\right)$ compared with MS-CNS $\left(\mathrm{MIC}_{50}=0.004 \mathrm{mg} / \mathrm{l} ; \mathrm{MIC}_{90}=0.015 \mathrm{mg} / \mathrm{l} ;\right.$ Table 7$)$. These differences were due to quinolone cross-resistance rather than methicillin-susceptibility, because levofloxacinsusceptible MR-CNS isolates had lower $\mathrm{MIC}_{90}$ values $\left(\mathrm{MIC}_{50}=0.004 \mathrm{mg} / \mathrm{l} ; \mathrm{MIC}_{90}=0.008 \mathrm{mg} / \mathrm{l}\right.$; Table 7).

Ozenoxacin had higher activity against methicillin-resistant CNS isolates and levofloxacin nonsusceptible CNS isolates $\left(\mathrm{MIC}_{50}=0.06 \mathrm{mg} / \mathrm{l}\right.$ and $\mathrm{MIC}_{90}=0.5 \mathrm{mg} / \mathrm{l}$ against both$)$ than mupirocin $\left(\mathrm{MIC}_{50}=0.25 \mathrm{mg} / \mathrm{l}\right.$ and $\mathrm{MIC}_{90}$ $>256 \mathrm{mg} / \mathrm{l}$ against both) and fusidic acid $\left(\mathrm{MIC}_{50}=0.12 \mathrm{mg} / \mathrm{l}\right.$ and $\mathrm{MIC}_{90}=0.16 \mathrm{mg} / \mathrm{l}$ against both; Table 7).

\section{Corynebacterium species (study 1)}

The range of ozenoxacin against Corynebacterium species was 0.008 to $\geq 4 \mathrm{mg} / \mathrm{l}$, with a $\mathrm{MIC}_{50}$ of $1 \mathrm{mg} / \mathrm{l}$. This activity was considerably greater than that of ciprofloxacin $\left(\mathrm{MIC}_{50}=8 \mathrm{mg} / \mathrm{l}\right)$ and levofloxacin $\left(\mathrm{MIC}_{50}=16 \mathrm{mg} / \mathrm{l}\right)$. Ozenoxacin $\left(\mathrm{MIC}_{90}\right.$ of $\left.\geq 4 \mathrm{mg} / \mathrm{l}\right)$ was less active than daptomycin and vancomycin $\left(\mathrm{MIC}_{90}=0.5 \mathrm{mg} / \mathrm{l}\right)$, and fusidic acid, linezolid and retapamulin $\left(\mathrm{MIC}_{90}=1 \mathrm{mg} / \mathrm{l}\right)$, against Corynebacterium species.

Ozenoxacin was significantly more potent $\left(\mathrm{MIC}_{90}=0.06 \mathrm{mg} / \mathrm{l}\right)$ than all reference compounds for levofloxacinsusceptible isolates of Corynebacterium species. By comparison, the rank order for reference compounds was gentamicin $\left(\mathrm{MIC}_{90}=0.25 \mathrm{mg} / \mathrm{l}\right)$; daptomycin and fusidic acid $\left(\mathrm{MIC}_{90}=0.5 \mathrm{mg} / \mathrm{l}\right)$; ciprofloxacin, levofloxacin, linezolid, neomycin, retapamulin and vancomycin $\left(\mathrm{MIC}_{90}=1 \mathrm{mg} / \mathrm{l}\right)$; cefuroxime and amoxicillin-clavulanate $\left(\mathrm{MIC}_{90}=8 \mathrm{mg} / \mathrm{l}\right)$; ceftriaxone and erythromycin $\left(\mathrm{MIC}_{90}=16 \mathrm{mg} / \mathrm{l}\right)$; penicillin and tetracycline $\left(\mathrm{MIC}_{90}=32 \mathrm{mg} / \mathrm{l}\right)$; mupirocin $\left(\mathrm{MIC}_{90}=128 \mathrm{mg} / \mathrm{l}\right)$ and clindamycin $\left(\mathrm{MIC}_{90} \geq 128 \mathrm{mg} / \mathrm{l}\right)$.

\section{Other isolates including Micrococcus \& Lactobacillus species (study 1)}

A small number of other strains were evaluated for ozenoxacin susceptibility in study 1 . The MIC range of ozenoxacin was $0.004-0.06 \mathrm{mg} / \mathrm{l}$ for Micrococcus spp. ( $\mathrm{n}=7)$; 0.008-0.25 mg/l for Group G Streptococcus spp. ( $\mathrm{n}=19) ; 0.008-0.03 \mathrm{mg} / \mathrm{l}$ for Group C Streptococcus spp. $(\mathrm{n}=9) ; 0.03-1 \mathrm{mg} / \mathrm{l}$ for Brevibacterium spp. $(\mathrm{n}=3)$; 


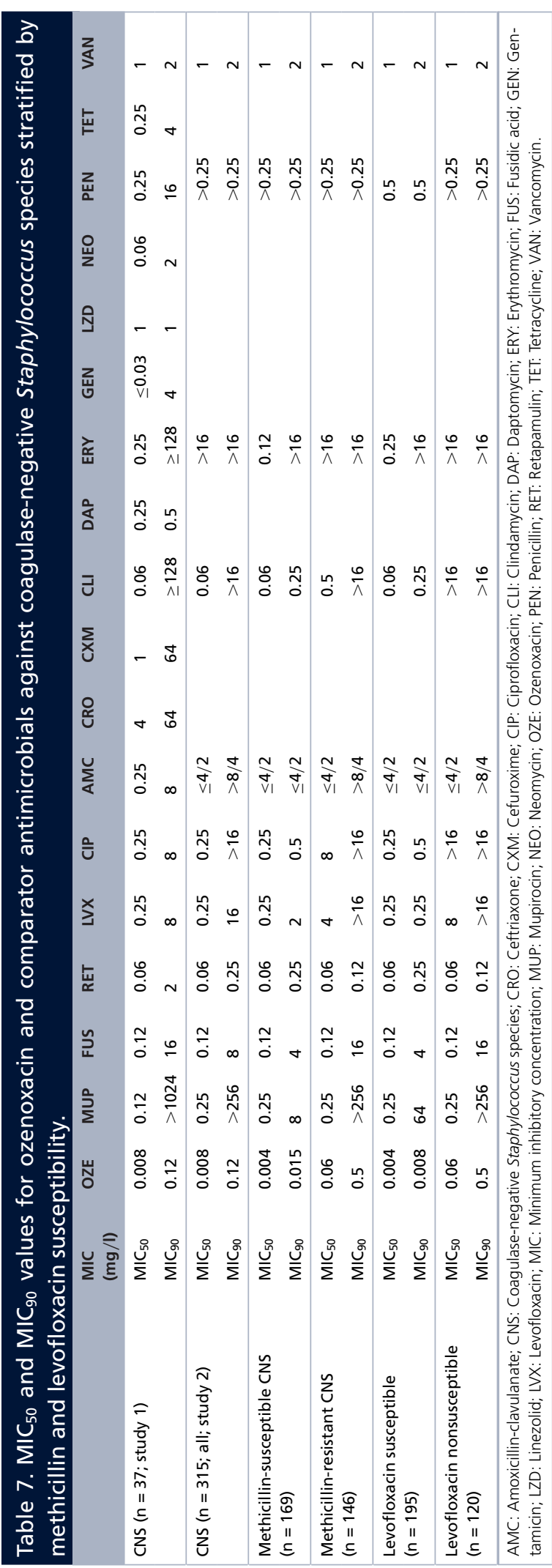


Table 8. Comparison of ozenoxacin $\mathrm{MIC}_{50}$ and $\mathrm{MIC}_{90}$ values in studies 1 and 2.

\begin{tabular}{|c|c|c|c|c|c|c|}
\hline \multirow[t]{2}{*}{ Microorganism isolate } & \multicolumn{3}{|c|}{ Study 1} & \multicolumn{3}{|c|}{ Study 2} \\
\hline & $\mathbf{n}$ & $\mathrm{MIC}_{50}$ & $\mathrm{MIC}_{90}$ & n & $\mathrm{MIC}_{50}$ & $\mathrm{MIC}_{90}$ \\
\hline S. aureus (all) & 486 & 0.004 & 0.25 & 504 & 0.002 & 0.06 \\
\hline MSSA & 247 & 0.004 & 0.004 & 279 & 0.002 & 0.004 \\
\hline MRSA & 239 & 0.12 & 0.25 & 225 & 0.004 & 0.12 \\
\hline Levofloxacin-susceptible S. aureus & 312 & 0.004 & 0.004 & 383 & 0.002 & 0.002 \\
\hline Levofloxacin-nonsusceptible $S$. aureus & 168 & 0.12 & 0.5 & 121 & 0.06 & 0.5 \\
\hline Levofloxacin-nonsusceptible MSSA & 14 & 0.12 & 1 & 18 & 0.06 & 0.12 \\
\hline Levofloxacin-susceptible MSSA & 231 & 0.004 & 0.004 & 261 & 0.002 & 0.002 \\
\hline Levofloxacin-susceptible MRSA & 81 & 0.004 & 0.008 & 122 & 0.002 & 0.002 \\
\hline Levofloxacin-nonsusceptible MRSA & 154 & 0.12 & 0.25 & 103 & 0.06 & 0.5 \\
\hline Streptococcus pyogenes & 217 & 0.03 & 0.06 & 124 & 0.008 & 0.015 \\
\hline Streptococcus agalactiae & 37 & 0.03 & 0.06 & 88 & 0.015 & 0.03 \\
\hline Streptococcus pneumoniae & 29 & 0.03 & 0.06 & & & \\
\hline S. epidermidis (all) & 190 & 0.06 & 1 & 195 & 0.008 & 0.25 \\
\hline MSSE & 64 & 0.008 & 0.12 & 86 & 0.004 & 0.03 \\
\hline MRSE & 126 & 0.12 & 2 & 109 & 0.06 & 0.5 \\
\hline Levofloxacin-susceptible S. epidermidis & 92 & 0.008 & 0.015 & 105 & 0.004 & 0.008 \\
\hline Levofloxacin-nonsusceptible S. epidermidis & 97 & 0.12 & 2 & 90 & 0.06 & 1 \\
\hline Levofloxacin-nonsusceptible, MSSE & 12 & 0.12 & 1 & 11 & 0.06 & 0.5 \\
\hline Levofloxacin-nonsusceptible, MRSE & 85 & 0.12 & 2 & 79 & 0.06 & 1 \\
\hline Levofloxacin-susceptible, MRSE & 41 & 0.008 & 0.015 & 30 & 0.004 & 0.008 \\
\hline Levofloxacin-susceptible, MSSE & 51 & 0.008 & 0.015 & 75 & 0.004 & 0.004 \\
\hline CNS (all) & 37 & 0.008 & 0.12 & 315 & 0.008 & 0.12 \\
\hline Methicillin-susceptible CNS & & & & 169 & 0.004 & 0.015 \\
\hline Methicillin-resistant CNS & & & & 146 & 0.06 & 0.5 \\
\hline Levofloxacin-susceptible CNS & & & & 195 & 0.004 & 0.008 \\
\hline Levofloxacin-nonsusceptible CNS & & & & 120 & 0.06 & 0.5 \\
\hline Corynebacterium species (all) & 52 & 1 & $\geq 4$ & & & \\
\hline Levofloxacin-resistant Corynebacterium species & 35 & 2 & $\geq 4$ & & & \\
\hline Levofloxacin-susceptible Corynebacterium species & 17 & 0.015 & 0.06 & & & \\
\hline
\end{tabular}

$0.03-2 \mathrm{mg} / 1$ for Enterococcus faecalis $(\mathrm{n}=2) ; 0.03-2 \mathrm{mg} / 1$ for Kocuria kristinae $(\mathrm{n}=1)$; and $0.015 \mathrm{mg} / \mathrm{l}$ for $R$. mucilaginosa $(\mathrm{n}=1)$.

\section{Comparison between study 1 \& study 2}

An overall summary comparing ozenoxacin $\mathrm{MIC}_{50}$ and $\mathrm{MIC}_{90}$ values in $S$. aureus, Streptococcus spp., S. epidermidis, coagulase-negative staphylococci and Corynebacterium spp. in both studies (Table 8) showed good general agreement.

\section{Discussion}

Clinical isolates were collected and analyzed for susceptibility or resistance to a panel of antimicrobial agents in two in vitro studies. The first collection of 1097 isolates from 49 centers was made during 2009-2010, and a later collection of 1031 clinical isolates at ten centers was made during 2014. S. aureus isolates predominated in both collections accounting for 44 and $49 \%$ of all isolates, respectively. S. aureus and S. epidermidis isolates were stratified by methicillin and levofloxacin resistance/susceptibility status. The antibacterial effects of ozenoxacin determined using $\mathrm{MIC}_{50}$ and $\mathrm{MIC}_{90}$ values were compared with 17 and ten antimicrobial agents, respectively. These included the topical agents mupirocin, fusidic acid and retapamulin, and also other antimicrobials for comparative activity against resistant and susceptible strains. 
Ozenoxacin was the most potent agent tested against all $S$. aureus isolates. At the $\mathrm{MIC}_{90}$ level, ozenoxacin $\left(\mathrm{MIC}_{90}=0.06 \mathrm{mg} / \mathrm{l}\right)$ was twofold more potent than retapamulin $\left(\mathrm{MIC}_{90}=0.12 \mathrm{mg} / \mathrm{l}\right)$, fourfold more potent than fusidic acid $\left(\mathrm{MIC}_{90}=0.25 \mathrm{mg} / \mathrm{l}\right)$, eightfold more active than mupirocin $\left(\mathrm{MIC}_{90}=0.5 \mathrm{mg} / \mathrm{l}\right)$ and at least 256 -fold more potent than erythromycin, clindamycin, ciprofloxacin or levofloxacin ( $\mathrm{MIC}_{90} \geq 16 \mathrm{mg} / 1$ for all; study 2 data).

Tested against levofloxacin nonsusceptible $S$. aureus isolates, ozenoxacin $\left(\mathrm{MIC}_{50} / 90,0.06 / 0.5 \mathrm{mg} / \mathrm{l}\right)$ was also the most potent compound. Only clindamycin had an $\mathrm{MIC}_{50}(0.06 \mathrm{mg} / \mathrm{l})$ equal to that of ozenoxacin. The remaining agents had higher $\mathrm{MIC}_{50}$ and $\mathrm{MIC}_{90}$ values than ozenoxacin (study 2 data).

Methicillin-resistant staphylococci had raised MICs to ozenoxacin, but this was due to quinolone cross-resistance rather than methicillin susceptibility, as levofloxacin-susceptible but methicillin-resistant staphylococci had an ozenoxacin $\mathrm{MIC}_{90}$ of 0.002 against $S$. aureus and $0.008 \mathrm{mg} / \mathrm{l}$ against coagulase-negative staphylococci (study 2 data). Compared with staphylococcal isolates with susceptibility to levofloxacin, ozenoxacin had lower activity when tested against levofloxacin nonsusceptible staphylococci with $\mathrm{MIC}_{50}$ and $\mathrm{MIC}_{90}$ values of 0.06 and $0.5 \mathrm{mg} / \mathrm{l}$, respectively (study 2 data). This clearly high intrinsic activity in levofloxacin nonsusceptible isolates was also addressed in a previous study in which the presence of GyrA and ParC amino acid substitutions were characterized [16]. Interestingly, only isolates with double mutations in both GyrA and ParC had ozenoxacin MIC values higher than $0.5 \mathrm{mg} / \mathrm{l}$. The percentage of $S$. aureus displaying MIC values more than $0.5 \mathrm{mg} / \mathrm{l}$ in study 2 was only $0.6 \%$.

Comparison of ozenoxacin $\mathrm{MIC}_{50}$ and $\mathrm{MIC}_{90}$ values for isolates collected in the two studies showed good general agreement and differences between study 1 and study 2 may reflect differences in the diversity of clinical isolates.

A previous study of ozenoxacin susceptibility performed in Japan reported $\mathrm{MIC}_{90}$ values for ozenoxacin against MSSA, MRSA and $S$. pyogenes isolates obtained from clinical cutaneous specimens of $\leq 0.06,4$ and $\leq 0.06 \mathrm{mg} / \mathrm{l}$, respectively. There was no difference between ozenoxacin $\mathrm{MIC}_{90}$ values for MSSA and $S$. pyogenes isolates obtained from adults and children, but the ozenoxacin $\mathrm{MIC}_{90}(0.12 \mu \mathrm{g} / \mathrm{ml})$ against pediatric MRSA isolates was 32-fold lower than that found for adult isolates [17]. This could be due to the impact of fluoroquinolone use in adults. In comparison, in the present studies, ozenoxacin $\mathrm{MIC}_{90}$ values were $0.004 \mathrm{mg} / \mathrm{l}$ in both studies 1 and 2 against MSSA; 0.25 and $0.12 \mathrm{mg} / \mathrm{l}$ in study 1 and study 2, respectively, against MRSA; and 0.06 and $0.015 \mathrm{mg} / \mathrm{l}$ in study 1 and study 2, respectively, against $S$. pyogenes.

A second Japanese study of the antimicrobial activity of ozenoxacin against isolates from patients with acne vulgaris reported $\mathrm{MIC}_{90}$ values of ozenoxacin against $S$. aureus, $S$. epidermidis and other coagulase-negative staphylococci of $\leq 0.06,0.125$ and $\leq 0.06 \mathrm{mg} / \mathrm{l}$, respectively [18]. In comparison, in the present studies, ozenoxacin MIC 90 values were 0.25 and $0.06 \mathrm{mg} / 1$ in study 1 and study 2, respectively, against $S$. aureus; 1 and $0.25 \mathrm{mg} / 1$ in study 1 and study 2, respectively, against $S$. epidermidis; and $0.12 \mathrm{mg} / \mathrm{l}$ in both studies 1 and 2 against coagulase-negative staphylococci. The $\mathrm{MIC}_{90}$ of ozenoxacin against Propionibacterium acnes was $\leq 0.06 \mathrm{mg} / \mathrm{l}$ in the Japanese study [18], but strains of this species were not included in the present study.

\section{Conclusion}

Ozenoxacin is a potent antimicrobial agent against both staphylococci and streptococci, irrespective of levofloxacin susceptibility status.

\section{Future perspective}

The in vitro spectrum of activity of ozenoxacin against staphylococci and streptococci, irrespective of methicillin or levofloxacin susceptibility, is mirrored by the efficacy of ozenoxacin in a clinical setting for the treatment of impetigo. Phase III trials showed that ozenoxacin produced a statistically significant superior microbiological response compared with placebo $[7,8]$, and had comparable efficacy to retapamulin but with a higher microbiological clearance rate [7]. These results support future ozenoxacin use and inclusion in impetigo guidelines.

Financial \& competing interests disclosure

The studies were supported by Ferrer Internacional, Barcelona, Spain. D Gargallo-Viola was an employee of Ferrer Internacional at the time the studies were performed. I Zsolt is a current employee of Ferrer Internacional. The authors have no other relevant affiliations or financial involvements with any organization or entity with a financial interest in or financial conflict with the subject matter or materials discussed in the manuscript apart from those disclosed.

Writing assistance was provided by Content Ed Net (Madrid, Spain) with funding provided by Ferrer International SA (Barcelona, Spain). 
Executive summary

- The in vitro activity of the anti-impetigo agent, ozenoxacin, and other antimicrobial agents against Gram-positive clinical isolates obtained from skin and soft tissue infections were compared.

- Isolates were collected in two studies: 1097 isolates from 49 centers during 2009-2010 in study 1 and 1031 isolates from ten centers during 2014 in study 2. The antibacterial effects of ozenoxacin were compared with 17 and ten antimicrobial agents in studies 1 and 2, respectively, by using MICs. Isolates were stratified by species and methicillin susceptibility/resistance and/or levofloxacin susceptibility/nonsusceptibility status.

- Comparison of ozenoxacin $\mathrm{MIC}_{50}$ and $\mathrm{MIC}_{90}$ values for isolates collected in both studies showed good general agreement. Overall, ozenoxacin was the most potent antimicrobial agent tested against staphylococci and streptococci. Ozenoxacin exhibited high in vitro activity against Staphylococcus aureus ( $\mathrm{MIC}_{90}=0.06 \mathrm{mg} / \mathrm{l}$; $\mathrm{n}=504$ ) and coagulase-negative staphylococci isolates in both studies (study 2 data: $\mathrm{MIC}_{90}=0.12 \mathrm{mg} / \mathrm{l} ; \mathrm{n}=315$ ). Ozenoxacin was also highly active against Streptococcus pyogenes $\left(\mathrm{MIC}_{90}=0.015 \mathrm{mg} / \mathrm{l} ; \mathrm{n}=217\right)$ and Streptococcus agalactiae ( $\mathrm{MIC}_{90}=0.03 \mathrm{mg} / \mathrm{l} ; \mathrm{n}=88$ ) isolates.

- In conclusion, ozenoxacin is a potent antimicrobial agent against staphylococci and streptococci, major pathogens involved in impetigo.

\section{References}

Papers of special note have been highlighted as: $\bullet$ of interest; $\bullet \bullet$ of considerable interest

1. Sladden MJ, Johnston GA. Common skin infections in children. BMJ 329, 95-99 (2004).

2. Hartman-Adams H, Banvard C, Juckett G. Impetigo: diagnosis and treatment. Am. Fam. Physician 90, 229-235 (2014).

3. Pereira LB. Impetigo - review. An. Bras. Dermatol. 89(2), 293-299 (2014).

4. George A, Rubin G. A systematic review and meta-analysis of treatments for impetigo. Br. J. Gen. Pract. 53(491), 480-487 (2003).

5. Koning S, van der Sande R, Verhagen AP et al. Interventions for impetigo. Cochrane Database Syst. Rev. 1, CD003261 (2012).

6. Yamakawa T, Mitsuyama J, Hayashi K. In vitro and in vivo antibacterial activity of T-3912, a novel non-fluorinated topical quinolone. J. Antimicrob. Chemother. 49(3), 455-465 (2002).

7. Gropper S, Albareda N, Chelius K et al. Ozenoxacin 1\% cream in the treatment of impetigo: a multicenter, randomized, placebo- and retapamulin-controlled clinical trial. Future Microbiol. 9(9), 1013-1023 (2014).

-. Phase III clinical trial showing the efficacy and safety of ozenoxacin $1 \%$ cream for the treatment of impetigo.

8. Albareda N, Zeichner J, Rosenberg $\mathrm{N}$ et al. A randomized vehicle-controlled trial to assess the efficacy, safety, and tolerability of ozenoxacin 1\% cream in 412 patients 2 months and older with impetigo. SKIN J. Cutan. Med. 1, s103 (2017).

- Phase III clinical trial showing the efficacy and safety of ozenoxacin in patients with impetigo.

9. Brauner A, Fridman O, Gefen O, Balaban NQ. Distinguishing between resistance, tolerance and persistence to antibiotic treatment. Nat. Rev. Microbiol. 14, 320-330 (2016).

10. Clinical and Laboratory Standards Institute (CLSI). Performance Standards for Antimicrobial Susceptibility Testing: Twentieth Informational Supplement. CLSI Document M100-S20. Wayne, PA, USA (2010).

11. Clinical and Laboratory Standards Institute (CLSI). Methods for Antimicrobial Dilution and Disk Susceptibility Testing of Infrequently Isolated or Fastidious Bacteria: Approved Guideline. CLSI Document M45-A. Wayne, PA, USA (2006).

12. European Committee on Antimicrobial Susceptibility Testing (EUCAST). Breakpoint tables for interpretation of MICs and zone diameters. Version 1.0, December 2009. www.eucast.org/clinical_breakpoints

13. British Society for Antimicrobial Chemotherapy. BSAC methods for antimicrobial susceptibility testing. Version 9.1, (2010). www.bsac.org.uk/Susceptibility+Testing/Breakpoints

14. Clinical and Laboratory Standards Institute (CLSI). Performance Standards for Antimicrobial Susceptibility Testing: Twentieth Informational Supplement. CLSI Document M100-S20, Wayne, PA, USA (2011).

15. Clinical and Laboratory Standards Institute (CLSI). Performance Standards for Antimicrobial Susceptibility Testing: Twentieth Informational Supplement. CLSI Document M100-S24, Wayne, PA, USA (2014).

16. López $\mathrm{Y}$, Tato M, Espinal $\mathrm{P}$ et al. In vitro activity of ozenoxacin against quinolone-susceptible and quinolone-resistant gram-positive bacteria. Antimicrob. Agents Chemother. 57, 6389-6392 (2013).

-. In vitro antibacterial activity of ozenoxacin against Gram-positive bacteria isolated from skin and soft tissue infections.

17. Kanayama S, Ikeda F, Okamoto K et al. In vitro antimicrobial activity of ozenoxacin against methicillin-susceptible Staphylococcus aureus, methicillin-resistant $S$. aureus and Streptococcus pyogenes isolated from clinical cutaneous specimens in Japan. J. Infect. Chemother. 22, 720-723 (2016).

•• In vitro antimicrobial activities of ozenoxacin against cutaneous isolates. 
18. Nakajima A, Ikeda F, Kanayama $S$ et al. Antimicrobial activities of ozenoxacin against isolates of propionibacteria and staphylococci from Japanese patients with acne vulgaris. J. Med. Microbiol. 65, 745-750 (2016).

-• In vitro antimicrobial activities of ozenoxacin against clinical isolates of propionibacteria and staphylococci. 
